\title{
Peterson's Deformations of Higher Dimensional Quadrics
}

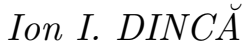

Faculty of Mathematics and Informatics, University of Bucharest, 14 Academiei Str., 010014, Bucharest, Romania

E-mail: dinca@gta.math.unibuc.ro

Received July 13, 2009, in final form January 16, 2010; Published online January 20, 2010 doi:10.3842/SIGMA.2010.006

\begin{abstract}
We provide the first explicit examples of deformations of higher dimensional quadrics: a straightforward generalization of Peterson's explicit 1-dimensional family of deformations in $\mathbb{C}^{3}$ of 2-dimensional general quadrics with common conjugate system given by the spherical coordinates on the complex sphere $\mathbb{S}^{2} \subset \mathbb{C}^{3}$ to an explicit $(n-1)$-dimensional family of deformations in $\mathbb{C}^{2 n-1}$ of $n$-dimensional general quadrics with common conjugate system given by the spherical coordinates on the complex sphere $\mathbb{S}^{n} \subset \mathbb{C}^{n+1}$ and nondegenerate joined second fundamental forms. It is then proven that this family is maximal.
\end{abstract}

Key words: Peterson's deformation; higher dimensional quadric; common conjugate system

2010 Mathematics Subject Classification: 53A07; 53B25; 35Q58

\section{Introduction}

The Russian mathematician Peterson was a student of Minding's, who in turn was interested in deformations (through bending) of surfaces ${ }^{1}$, but unfortunately most of his works (including his independent discovery of the Codazzi-Mainardi equations and of the Gauß-Bonnet theorem) were made known to Western Europe mainly after they were translated in 1905 from Russian to French (as is the case with his deformations of quadrics [7], originally published in 1883 in Russian). Peterson's work on deformations of general quadrics preceded that of Bianchi, Calapso, Darboux, Guichard and Țiţeica's from the years 1899-1906 by two decades; in particular Peterson's 1-dimensional family of deformations of surfaces admitting a common conjugate system $(u, v)$ (that is the second fundamental form is missing mixed terms $d u \odot d v$ ) are associates (a notion naturally appearing in the infinitesimal deformation problem) to Bianchi's 1-dimensional family of surfaces satisfying $(\log K)_{u v}=0$ in the common asymptotic coordinates $(u, v), K$ being the Gauß curvature (see Bianchi [2, Vol. 2, $\S \S 294,295]$ ).

The work of these illustrious geometers on deformations in $\mathbb{C}^{3}$ of quadrics in $\mathbb{C}^{3}$ (there is no other class of surfaces for which an interesting theory of deformation has been built) is one of the crowning achievements of the golden age of classical geometry of surfaces and at the same time it opened new areas of research (such as affine and projective differential geometry) continued later by other illustrious geometers (Blaschke, Cartan, etc.).

Peterson's 1-dimensional family of deformations of 2-dimensional quadrics is obtained by imposing an ansatz naturally appearing from a geometric point of view, namely the constraint that the common conjugate system of curves is given by intersection with planes through an axis and tangent cones centered on that axis; thus this result of Koenigs (see Darboux [5, $\S \S 91-101]$ ) was again (at least when the cones are tangent along plane curves) previously known to Peterson. Note also that Calapso in [3] has put Bianchi's Bäcklund transformation of

\footnotetext{
${ }^{1}$ See Peterson's biography at http://www-history.mcs.st-and.ac.uk/Biographies/Peterson.html.
} 
deformations in $\mathbb{C}^{3}$ of general 2-dimensional quadrics with center in terms of common conjugate systems (the condition that the conjugate system on a 2-dimensional quadric is a conjugate system on one of its deformations in $\mathbb{C}^{3}$ was known to Calapso for a decade, but the Bäcklund transformation for general quadrics eluded Calapso since the common conjugate system was best suited for this transformation only at the analytic level).

Although this is the original approach Peterson used to find his deformations of quadrics, other features of his approach will make it amenable to higher dimensional generalizations, namely the warping of linear element (the warping of the linear element of a plane curve to get the linear element of a surface of revolution $\left(d\left(f \cos \left(u^{1}\right)\right)\right)^{2}+\left(d\left(f \sin \left(u^{1}\right)\right)\right)^{2}=(d f)^{2}+f^{2}\left(d u^{1}\right)^{2}$ for $f=f\left(u^{2}\right)$ is such an example) and separation of variables; post-priori the common conjugate system property may be given a geometric explanation analogous to that in dimension 3 .

In 1919-1920 Cartan has shown in [4] (using mostly projective arguments and his exterior differential systems in involution and exteriorly orthogonal forms tools) that space forms of dimension $n$ admit rich families of deformations (depending on $n(n-1)$ functions of one variable) in surrounding $(2 n-1)$-dimensional space forms, that such deformations admit lines of curvature (given by a canonical form of exteriorly orthogonal forms; thus they have flat normal bundle; since the lines of curvature on $n$-dimensional space forms (when they are considered by definition as quadrics in surrounding $(n+1)$-dimensional space forms) are undetermined, the lines of curvature on the deformation and their corresponding curves on the quadric provide the common conjugate system) and that the co-dimension $(n-1)$ cannot be lowered without obtaining rigidity as the deformation being the defining quadric.

In 1983 Berger, Bryant and Griffiths [1] proved (including by use of tools from algebraic geometry) in particular that Cartan's essentially projective arguments (including the exterior part of his exteriorly orthogonal forms tool) can be used to generalize his results to $n$-dimensional general quadrics with positive definite linear element (thus they can appear as quadrics in $\mathbb{R}^{n+1}$ or as space-like quadrics in $\mathbb{R}^{n} \times(i \mathbb{R})$ ) admitting rich families of deformations (depending on $n(n-1)$ functions of one variable) in surrounding Euclidean space $\mathbb{R}^{2 n-1}$, that the co-dimension $(n-1)$ cannot be lowered without obtaining rigidity as the deformation being the defining quadric and that quadrics are the only Riemannian $n$-dimensional manifolds that admit a family of deformations in $\mathbb{R}^{2 n-1}$ as rich as possible for which the exteriorly orthogonal forms tool (naturally appearing from the Gauß equations) can be applied.

Although Berger, Bryant and Griffiths [1] do not explicitly state the common conjugate system property (which together with the non-degenerate joined second fundamental forms assumption provides a tool similar to the canonical form of exteriorly orthogonal forms), this will turn out to be the correct tool of differential geometry needed to attack the deformation problem for higher dimensional quadrics; also at least for diagonal quadrics without center Peterson's deformations of higher dimensional quadrics will turn out to be amenable to explicit computations of their Bäcklund transformation ${ }^{2}$.

All computations are local and assumed to be valid on their open domain of validity without further details; all functions have the assumed order of differentiability and are assumed to be invertible, non-zero, etc when required (for all practical purposes we can assume all functions to be analytic).

Here we have the two main theorems concerning the $(n-1)$-dimensional family of deformations of higher dimensional general quadrics and respectively its maximality:

Theorem 1. The quadric

$$
\sum_{j=0}^{n} \frac{\left(x_{j}\right)^{2}}{a_{j}}=1, \quad a_{j} \in \mathbb{C}^{*}
$$

\footnotetext{
${ }^{2}$ See Dincă I.I., Bianchi's Bäcklund transformation for higher dimensional quadrics, arXiv:0808.2007.
} 
distinct parameterized with the conjugate system $\left(u^{1}, \ldots, u^{n}\right) \subset \mathbb{C}^{n}$ given by the spherical coordinates on the unit sphere $\mathbb{S}^{n} \subset \mathbb{C}^{n+1}$ :

$$
\mathcal{X}=\sqrt{a_{0}} \mathbf{C}_{0} e_{0}+\sum_{k=1}^{n} \sqrt{a_{k}} \mathbf{C}_{k} \sin \left(u^{k}\right) e_{k}, \quad \mathbf{C}_{k}:=\prod_{j=k+1}^{n} \cos \left(u^{j}\right)
$$

and the sub-manifold

$$
\mathcal{X}_{\mathbf{z}}=\sum_{k=1}^{n-1} \mathbf{C}_{k} f_{k}\left(\mathbf{z}, u^{k}\right)\left(\cos \left(g_{k}\left(\mathbf{z}, u^{k}\right)\right) e_{2 k-2}+\sin \left(g_{k}\left(\mathbf{z}, u^{k}\right)\right) e_{2 k-1}\right)+h\left(\mathbf{z}, u^{n}\right) e_{2 n-2}
$$

of $\mathbb{C}^{2 n-1}$ depending on the parameters $\mathbf{z}=\left(z_{1}, z_{2}, \ldots, z_{n-1}\right) \in \mathbb{C}^{n-1}, z_{0}:=1$ and with

$$
\begin{aligned}
& f_{k}\left(z_{k-1}, z_{k}, u^{k}\right):=\sqrt{\left(z_{k-1}-z_{k}\right) a_{0}+\left(a_{k}-z_{k-1} a_{0}\right) \sin ^{2}\left(u^{k}\right)}, \quad k=1, \ldots, n-1, \\
& g_{k}\left(z_{k-1}, z_{k}, u^{k}\right):=\int_{0}^{u^{k}} \frac{\sqrt{\left(z_{k-1}-z_{k}\right) a_{0} a_{k}+\left(a_{k}-z_{k-1} a_{0}\right) z_{k} a_{0} \sin ^{2}(t)}}{\left(z_{k-1}-z_{k}\right) a_{0}+\left(a_{k}-z_{k-1} a_{0}\right) \sin ^{2}(t)} d t, \\
& h\left(z_{n-1}, u^{n}\right):=\int_{0}^{u^{n}} \sqrt{a_{n}-\left(a_{n}-z_{n-1} a_{0}\right) \sin ^{2}(t)} d t
\end{aligned}
$$

have the same linear element $|d \mathcal{X}|^{2}=\left|d \mathcal{X}_{\mathbf{z}}\right|^{2}$. For $z_{1}=\cdots=z_{n-1}=0$ we get $g_{2}=\cdots=g_{n-1}=0$, $\mathcal{X}=\mathcal{X}_{\mathbf{0}}$ with $\mathbb{C}^{n+1} \hookrightarrow \mathbb{C}^{2 n-1}$ as $\left(x_{0}, x_{1}, \ldots, x_{n}\right) \mapsto\left(x_{0}, x_{1}, x_{2}, 0, x_{3}, 0, \ldots, x_{n-1}, 0, x_{n}\right)$. For $z_{1}=\cdots=z_{n-1}=1$ we get $\mathcal{X}_{\mathbf{1}}=\left(\mathbf{x}_{0}, \ldots, \mathbf{x}_{2 n-2}\right)$ given by Peterson's formulae

$$
\begin{aligned}
& \sqrt{\left(\mathbf{x}_{2 k-2}\right)^{2}+\left(\mathbf{x}_{2 k-1}\right)^{2}}=\sqrt{a_{k}-a_{0}} \mathbf{C}_{k} \sin \left(u^{k}\right), \\
& \tan ^{-1}\left(\frac{\mathbf{x}_{2 k-1}}{\mathbf{x}_{2 k-2}}\right)=\frac{\sqrt{a_{0}}}{\sqrt{a_{k}-a_{0}}} \tanh ^{-1}\left(\cos \left(u^{k}\right)\right), \quad k=1, \ldots, n-1, \\
& \mathbf{x}_{2 n-2}=\int_{0}^{u^{n}} \sqrt{a_{n}-\left(a_{n}-a_{0}\right) \sin ^{2}(t)} d t .
\end{aligned}
$$

Moreover $\left(u^{1}, \ldots, u^{n}\right)$ form a conjugate system on $\mathcal{X}_{\mathbf{z}}$ with non-degenerate joined second fundamental forms (that is $\left[\begin{array}{lll}d^{2} \mathcal{X}^{T} N & d^{2} \mathcal{X}_{\mathbf{z}}^{T} N_{\mathbf{z}}\end{array}\right]$ is a symmetric quadratic $\mathbb{C}^{n}$-valued form which contains only $\left(d u^{j}\right)^{2}$ terms for $N$ normal field of $\mathcal{X}$ and $N_{\mathbf{z}}=\left[\begin{array}{llll}N_{1} & \ldots & N_{n-1}\end{array}\right]$ normal frame of $\mathcal{X}_{\mathbf{z}}$ and the dimension $n$ cannot be lowered for $\mathbf{z}$ in an open dense set).

Theorem 2. For $x \subset \mathbb{C}^{2 n-1}$ deformation of the quadric $x_{0} \subset \mathbb{C}^{n+1}$ (that is $|d x|^{2}=\left|d x_{0}\right|^{2}$ ) with $n \geq 3,\left(u^{1}, \ldots, u^{n}\right)$ common conjugate system and non-degenerate joined second fundamental forms, $N_{0}^{T} d^{2} x_{0}=: \sum_{j=1}^{n} h_{j}^{0}\left(d u^{j}\right)^{2}$ second fundamental form of $x_{0}$ we have $\Gamma_{j k}^{l}=0$ for $j, k, l$ distinct and such deformations are in bijective correspondence with solutions $\left\{\mathbf{a}_{j}\right\}_{j=1, \ldots, n} \subset \mathbb{C}^{*}$ of the differential system $\partial_{u^{k}} \log \mathbf{a}_{j}=\Gamma_{j k}^{j}, j \neq k, \sum_{j=1}^{n} \frac{\left(h_{j}^{0}\right)^{2}}{\mathbf{a}_{j}^{2}}+1=0$. In particular this implies that for $\left(u^{1}, \ldots, u^{n}\right)$ being the conjugate system given by spherical coordinates on $\mathbb{S}^{n} \subset \mathbb{C}^{n+1}$ the above explicit $(n-1)$-dimensional family of deformations $\mathcal{X}_{\mathbf{z}}$ is maximal.

The remaining part of this paper is organized as follows: in Section 2 we shall recall Peterson's deformations of quadrics; the proof of Theorem 1 appears in Sections 3, 4 and the proof of Theorem 2 appears in Sections 5, 6. 


\section{Peterson's deformations of quadrics}

Although Peterson [7] discusses all types of quadrics in the complexified Euclidean space

$$
\left(\mathbb{C}^{3},\langle\cdot, \cdot\rangle\right), \quad\langle x, y\rangle:=x^{T} y, \quad|x|^{2}:=x^{T} x \quad \text { for } \quad x, y \in \mathbb{C}^{3}
$$

and their totally real cases, we shall only discuss quadrics of the type $\sum_{j=0}^{2} \frac{\left(x_{j}\right)^{2}}{a_{j}}=1, a_{j} \in \mathbb{C}^{*}$ distinct, since the remaining cases of quadrics should follow by similar computations. Their totally real cases (that is $\left(x_{j}\right)^{2}, a_{j} \in \mathbb{R}$ ) are discussed in detail in Peterson [7], so we shall not insist on this aspect.

Remark 1. It is less known since the classical times that there are many types of quadrics from a complex metric point of view, each coming with its own totally real cases (real valued (in)definite linear element); among these quadrics for example the quadric $\left(x_{0}-i x_{1}\right) x_{2}-\left(x_{0}+\right.$ $\left.i x_{1}\right)=0$ is rigidly applicable (isometric) to all quadrics of its confocal family and to all its homothetic quadrics. It is Peterson who first introduced the idea of ideal applicability (for example a real surface may be applicable to a totally real space-like surface $\subset \mathbb{R}^{2} \times(i \mathbb{R})$ of a complexified real ellipsoid, so it is ideally applicable on the real ellipsoid).

With $\left\{e_{j}\right\}_{j=0,1,2}, e_{j}^{T} e_{k}=\delta_{j k}$ the standard basis of $\mathbb{C}^{3}$ and the functions $f=f\left(z, u^{1}\right), g=$ $g\left(z, u^{1}\right), h=h\left(z, u^{2}\right)$ depending on the parameter(s) $z=\left(z_{1}, z_{2}, \ldots\right)$ to be determined later we have the surfaces

$$
\mathcal{X}_{z}:=\cos \left(u^{2}\right) f\left(z, u^{1}\right)\left(\cos \left(g\left(z, u^{1}\right)\right) e_{0}+\sin \left(g\left(z, u^{1}\right)\right) e_{1}\right)+h\left(z, u^{2}\right) e_{2} .
$$

Note that the fields $\left.\partial_{u^{1}} \mathcal{X}_{z}\right|_{u^{1}=\text { const }},\left.\partial_{u^{2}} \mathcal{X}_{z}\right|_{u^{2}=\text { const }}$ generate developables (cylinders with generators perpendicular on the third axis and cones centered on the third axis), so $\left(u^{1}, u^{2}\right)$ is a conjugate system on $\mathcal{X}_{z}$ for every $z$; in fact all surfaces have conjugate systems arising this way and can be parameterized as

$$
x=f\left(u^{1}, u^{2}\right)\left(\cos \left(u^{1}\right) e_{0}+\sin \left(u^{1}\right) e_{1}\right)+g\left(u^{1}, u^{2}\right) e_{2}, \quad \partial_{u^{1}}\left(\partial_{u^{2}}\left(\frac{g}{f}\right) / \partial_{u^{2}}\left(\frac{1}{f}\right)\right)=0 .
$$

The quadric $\sum_{j=0}^{2} \frac{\left(x_{j}\right)^{2}}{a_{j}}=1$ is parameterized by the spherical coordinates

$$
\mathcal{X}=\sqrt{a_{0}} \cos \left(u^{2}\right) \cos \left(u^{1}\right) e_{0}+\sqrt{a_{1}} \cos \left(u^{2}\right) \sin \left(u^{1}\right) e_{1}+\sqrt{a_{2}} \sin \left(u^{2}\right) e_{2} .
$$

We have

$$
\begin{aligned}
\left|d \mathcal{X}_{z}\right|^{2}= & \cos ^{2}\left(u^{2}\right)\left(f^{\prime 2}\left(z, u^{1}\right)+f^{2}\left(z, u^{1}\right) g^{\prime 2}\left(z, u^{1}\right)\right)\left(d u^{1}\right)^{2} \\
& +\frac{1}{2} d\left(\cos ^{2}\left(u^{2}\right)\right) d\left(f^{2}\left(z, u^{1}\right)\right)+\left(f^{2}\left(z, u^{1}\right) \sin ^{2}\left(u^{2}\right)+h^{\prime 2}\left(z, u^{2}\right)\right)\left(d u^{2}\right)^{2}, \\
|d \mathcal{X}|^{2}= & \cos ^{2}\left(u^{2}\right)\left(a_{1}-\left(a_{1}-a_{0}\right) \sin ^{2}\left(u^{1}\right)\right)\left(d u^{1}\right)^{2} \\
& +\frac{1}{2} d\left(\cos ^{2}\left(u^{2}\right)\right) d\left(a_{0}+\left(a_{1}-a_{0}\right) \sin ^{2}\left(u^{1}\right)\right) \\
& +\left(a_{2}-\left(a_{2}-a_{0}-\left(a_{1}-a_{0}\right) \sin ^{2}\left(u^{1}\right)\right) \sin ^{2}\left(u^{2}\right)\right)\left(d u^{2}\right)^{2} .
\end{aligned}
$$

Thus the condition $\left|d \mathcal{X}_{z}\right|^{2}=|d \mathcal{X}|^{2}$ becomes

$$
\begin{aligned}
& f^{2}\left(z, u^{1}\right)+\left(a_{2}-a_{0}-\left(a_{1}-a_{0}\right) \sin ^{2}\left(u^{1}\right)\right)=\mathrm{const}=\frac{a_{2}-h^{\prime 2}\left(z, u^{2}\right)}{\sin ^{2}\left(u^{2}\right)}, \\
& f^{\prime 2}\left(z, u^{1}\right)+f^{2}\left(z, u^{1}\right) g^{\prime 2}\left(z, u^{1}\right)=a_{1}-\left(a_{1}-a_{0}\right) \sin ^{2}\left(u^{1}\right),
\end{aligned}
$$


from where we get

$$
\begin{aligned}
h\left(z_{1}, u^{2}\right) & :=\int_{0}^{u^{2}} \sqrt{a_{2}-\left(a_{2}-z_{1} a_{0}\right) \sin ^{2}(t)} d t, \\
f\left(z_{1}, u^{1}\right) & :=\sqrt{\left(1-z_{1}\right) a_{0}+\left(a_{1}-a_{0}\right) \sin ^{2}\left(u^{1}\right)}, \\
g\left(z_{1}, u^{1}\right) & :=\int_{0}^{u^{1}} \frac{\sqrt{\left(1-z_{1}\right) a_{0} a_{1}+\left(a_{1}-a_{0}\right) z_{1} a_{0} \sin ^{2}(t)}}{\left(1-z_{1}\right) a_{0}+\left(a_{1}-a_{0}\right) \sin ^{2}(t)} d t .
\end{aligned}
$$

Note that

$$
f\left(0, u^{1}\right) \cos \left(g\left(0, u^{1}\right)\right)=\sqrt{a_{0}} \cos \left(u^{1}\right), \quad f\left(0, u^{1}\right) \sin \left(g\left(0, u^{1}\right)\right)=\sqrt{a_{1}} \sin \left(u^{1}\right),
$$

(we assume simplifications of the form $\sqrt{a} \sqrt{b} \simeq \sqrt{a b}$ with $\sqrt{\cdot}$ having the usual definition $\sqrt{r e^{i \theta}}:=$

$\sqrt{r} e^{\frac{i \theta}{2}}, r>0,-\pi<\theta \leq \pi$, since the possible signs are accounted by symmetries in the principal planes for quadrics and disappear at the level of the linear element for their deformations), so $\mathcal{X}=\mathcal{X}_{0}$.

The coordinates $\mathbf{x}_{0}, \mathbf{x}_{1}, \mathbf{x}_{2}$ of $\mathcal{X}_{1}$ satisfy (modulo a sign at the second formula) Peterson's formulae:

$$
\begin{aligned}
& \sqrt{\left(\mathbf{x}_{0}\right)^{2}+\left(\mathbf{x}_{1}\right)^{2}}=\sqrt{a_{1}-a_{0}} \cos \left(u^{2}\right) \sin \left(u^{1}\right), \\
& \tan ^{-1}\left(\frac{\mathbf{x}_{1}}{\mathbf{x}_{0}}\right)=\frac{\sqrt{a_{0}}}{\sqrt{a_{1}-a_{0}}} \tanh ^{-1}\left(\cos \left(u^{1}\right)\right), \\
& \mathbf{x}_{2}=\int_{0}^{u^{2}} \sqrt{a_{2}-\left(a_{2}-a_{0}\right) \sin ^{2}(t)} d t .
\end{aligned}
$$

More generally

$$
\begin{aligned}
h\left(z_{1}, u^{2}\right) & :=\int_{0}^{u^{2}} \sqrt{h^{\prime 2}(t)-z_{1} \sin ^{2}(t)} d t, \\
f\left(z_{1}, u^{1}\right) & :=\sqrt{z_{1}+f^{2}\left(u^{1}\right)}, \\
g\left(z_{1}, u^{1}\right) & :=\int_{0}^{u^{1}} \frac{\sqrt{z_{1}\left(f^{\prime 2}(t)+f^{2}(t) g^{\prime 2}(t)\right)+f^{4}(t) g^{\prime 2}(t)}}{z_{1}+f^{2}(t)} d t
\end{aligned}
$$

give Peterson's 1-dimensional family of deformations (3) with common conjugate system $\left(u^{1}, u^{2}\right)$.

\section{Peterson's deformations of higher dimensional quadrics}

Again we shall discuss only the case of quadrics with center and having distinct eigenvalues of the quadratic part defining the quadric, without insisting on totally real cases and deformations (when the linear elements are real valued).

Remark 2. A metric classification of all (totally real) quadrics in $\mathbb{C}^{n+1}$ requires the notion of symmetric Jordan canonical form of a symmetric complex matrix (see, e.g. [6]). The symmetric Jordan blocks are:

$$
\begin{aligned}
& J_{1}:=0=0_{1,1} \in \mathbf{M}_{1}(\mathbb{C}), \quad J_{2}:=f_{1} f_{1}^{T} \in \mathbf{M}_{2}(\mathbb{C}), \quad J_{3}:=f_{1} e_{3}^{T}+e_{3} f_{1}^{T} \in \mathbf{M}_{3}(\mathbb{C}), \\
& J_{4}:=f_{1} \bar{f}_{2}^{T}+f_{2} f_{2}^{T}+\bar{f}_{2} f_{1}^{T} \in \mathbf{M}_{4}(\mathbb{C}), \quad J_{5}:=f_{1} \bar{f}_{2}^{T}+f_{2} e_{5}^{T}+e_{5} f_{2}^{T}+\bar{f}_{2} f_{1}^{T} \in \mathbf{M}_{5}(\mathbb{C}), \\
& J_{6}:=f_{1} \bar{f}_{2}^{T}+f_{2} \bar{f}_{3}^{T}+f_{3} f_{3}^{T}+\bar{f}_{3} f_{2}^{T}+\bar{f}_{2} f_{1}^{T} \in \mathbf{M}_{6}(\mathbb{C}),
\end{aligned}
$$


etc., where $f_{j}:=\frac{e_{2 j-1}-i e_{2 j}}{\sqrt{2}}$ are the standard isotropic vectors (at least the blocks $J_{2}, J_{3}$ were known to the classical geometers). Any symmetric complex matrix can be brought via conjugation with a complex rotation to the symmetric Jordan canonical form, that is a matrix block decomposition with blocks of the form $a_{j} I_{p}+J_{p}$; totally real quadrics are obtained for eigenvalues $a_{j}$ of the quadratic part defining the quadric being real or coming in complex conjugate pairs $a_{j}, \bar{a}_{j}$ with subjacent symmetric Jordan blocks of same dimension $p$.

Consider the quadric $\sum_{j=0}^{n} \frac{\left(x_{j}\right)^{2}}{a_{j}}=1, a_{j} \in \mathbb{C}^{*}$ distinct with parametrization given by the spherical coordinates on the unit sphere $\mathbb{S}^{n} \subset \mathbb{C}^{n+1}$

$$
\mathcal{X}=\sqrt{a_{0}} \mathbf{C}_{0} e_{0}+\sum_{k=1}^{n} \sqrt{a_{k}} \mathbf{C}_{k} \sin \left(u^{k}\right) e_{k}, \quad \mathbf{C}_{k}:=\prod_{j=k+1}^{n} \cos \left(u^{j}\right) .
$$

The correct generalization of (3) allows us to build Peterson's deformations of higher dimensional quadrics. With an eye to the case $n=2$ we make the natural ansatz

$$
\mathcal{X}_{\mathbf{z}}=\sum_{k=1}^{n-1} \mathbf{C}_{k} f_{k}\left(\mathbf{z}, u^{k}\right)\left(\cos \left(g_{k}\left(\mathbf{z}, u^{k}\right)\right) e_{2 k-2}+\sin \left(g_{k}\left(\mathbf{z}, u^{k}\right)\right) e_{2 k-1}\right)+h\left(\mathbf{z}, u^{n}\right) e_{2 n-2}
$$

with the parameter(s) $\mathbf{z}=\left(z_{1}, z_{2}, \ldots\right)$ to be determined later.

We have

$$
\begin{aligned}
\left|d \mathcal{X}_{\mathbf{z}}\right|^{2}= & \sum_{k=1}^{n-1}\left[\mathbf{C}_{k}^{2}\left(f_{k}^{\prime 2}\left(\mathbf{z}, u^{k}\right)+f_{k}^{2}\left(\mathbf{z}, u^{k}\right) g_{k}^{\prime 2}\left(\mathbf{z}, u^{k}\right)\right)\left(d u^{k}\right)^{2}+\frac{1}{2} d\left(\mathbf{C}_{k}^{2}\right) d\left(f_{k}^{2}\left(\mathbf{z}, u^{k}\right)\right)\right. \\
& \left.+f_{k}^{2}\left(\mathbf{z}, u^{k}\right)\left(d \mathbf{C}_{k}\right)^{2}\right]+h^{\prime 2}\left(\mathbf{z}, u^{n}\right)\left(d u^{n}\right)^{2} \\
|d \mathcal{X}|^{2}= & a_{0}\left(d \mathbf{C}_{0}\right)^{2}+\sum_{k=1}^{n} a_{k}\left(d\left(\mathbf{C}_{k} \sin \left(u^{k}\right)\right)\right)^{2} .
\end{aligned}
$$

Comparing the coefficients of $\left(d u^{n}\right)^{2}$ from $\left|d \mathcal{X}_{\mathbf{z}}\right|^{2}=|d \mathcal{X}|^{2}$ we get

$$
\begin{aligned}
& \frac{1}{\cos ^{2}\left(u^{n}\right)}\left[\mathbf{C}_{1}^{2}\left(f_{1}^{2}\left(\mathbf{z}, u^{1}\right)-a_{0}-\left(a_{1}-a_{0}\right) \sin ^{2}\left(u^{1}\right)\right)+\sum_{k=2}^{n-1} \mathbf{C}_{k}^{2}\left(f_{k}^{2}\left(\mathbf{z}, u^{k}\right)-a_{k} \sin ^{2}\left(u^{k}\right)\right)\right] \\
& =\text { const }=\frac{a_{n} \cos ^{2}\left(u^{n}\right)-h^{\prime 2}\left(\mathbf{z}, u^{n}\right)}{\sin ^{2}\left(u^{n}\right)}
\end{aligned}
$$

from where we get with $z_{0}:=1$ :

$$
\begin{aligned}
& f_{k}^{2}\left(z_{k-1}, z_{k}, u^{k}\right):=\left(z_{k-1}-z_{k}\right) a_{0}+\left(a_{k}-z_{k-1} a_{0}\right) \sin ^{2}\left(u^{k}\right), \quad k=1, \ldots, n-1, \\
& h^{\prime 2}\left(z_{n-1}, u^{n}\right):=a_{n}-\left(a_{n}-z_{n-1} a_{0}\right) \sin ^{2}\left(u^{n}\right) .
\end{aligned}
$$

Now we have

$$
\begin{gathered}
\left(d \mathbf{C}_{0}\right)^{2}=\sum_{k=1}^{n-1}\left[z_{k-1}\left(d \mathbf{C}_{k-1}\right)^{2}-z_{k}\left(d \mathbf{C}_{k}\right)^{2}\right]+z_{n-1}\left(d \mathbf{C}_{n-1}\right)^{2}=\sum_{k=1}^{n-1}\left[z _ { k - 1 } \left(\mathbf{C}_{k}^{2} \sin ^{2}\left(u^{k}\right)\left(d u^{k}\right)^{2}\right.\right. \\
\left.\left.-\frac{1}{2} d\left(\mathbf{C}_{k}^{2}\right) d\left(\sin ^{2}\left(u^{k}\right)\right)+\cos ^{2}\left(u^{k}\right)\left(d \mathbf{C}_{k}\right)^{2}\right)-z_{k}\left(d \mathbf{C}_{k}\right)^{2}\right]+z_{n-1}\left(d \mathbf{C}_{n-1}\right)^{2}, \\
\left(d\left(\mathbf{C}_{k} \sin \left(u^{k}\right)\right)\right)^{2}=\mathbf{C}_{k}^{2} \cos ^{2}\left(u^{k}\right)\left(d u^{k}\right)^{2}+\frac{1}{2} d\left(\mathbf{C}_{k}^{2}\right) d\left(\sin ^{2}\left(u^{k}\right)\right)+\sin ^{2}\left(u^{k}\right)\left(d \mathbf{C}_{k}\right)^{2},
\end{gathered}
$$


so

$$
\begin{aligned}
&|d \mathcal{X}|^{2}= \sum_{k=1}^{n-1}\left[\mathbf{C}_{k}^{2}\left(a_{k}-\left(a_{k}-z_{k-1} a_{0}\right) \sin ^{2}\left(u^{k}\right)\right)\left(d u^{k}\right)^{2}+\frac{1}{2}\left(a_{k}-z_{k-1} a_{0}\right) d\left(\mathbf{C}_{k}^{2}\right) d\left(\sin ^{2}\left(u^{k}\right)\right)\right. \\
&\left.+\left(\left(z_{k-1}-z_{k}\right) a_{0}+\left(a_{k}-z_{k-1} a_{0}\right) \sin ^{2}\left(u^{k}\right)\right)\left(d \mathbf{C}_{k}\right)^{2}\right] \\
&+\left(a_{n}-\left(a_{n}-z_{n-1} a_{0}\right) \sin ^{2}\left(u^{n}\right)\right)\left(d u^{n}\right)^{2} \\
& 0=\left|d \mathcal{X}_{\mathbf{z}}\right|^{2}-|d \mathcal{X}|^{2}=\sum_{k=1}^{n-1} \mathbf{C}_{k}^{2}\left(f_{k}^{\prime 2}\left(\mathbf{z}, u^{k}\right)+f_{k}^{2}\left(\mathbf{z}, u^{k}\right) g_{k}^{\prime 2}\left(\mathbf{z}, u^{k}\right)\right. \\
&\left.-a_{k}+\left(a_{k}-z_{k-1} a_{0}\right) \sin ^{2}\left(u^{k}\right)\right)\left(d u^{k}\right)^{2}
\end{aligned}
$$

so we finally get (1).

For $z_{1}=z_{2}=\cdots=z_{n-1}=0$ we get $g_{2}=\cdots=g_{n-1}=0$ and using (5) we get $\mathcal{X}=\mathcal{X}_{\mathbf{0}}$ with $\mathbb{C}^{n+1} \hookrightarrow \mathbb{C}^{2 n-1}$ as $\left(x_{0}, x_{1}, \ldots, x_{n}\right) \mapsto\left(x_{0}, x_{1}, x_{2}, 0, x_{3}, 0, \ldots, x_{n-1}, 0, x_{n}\right)$.

For $z_{1}=z_{2}=\cdots=z_{n-1}=1$ we get $\mathcal{X}_{1}=\left(\mathbf{x}_{0}, \ldots, \mathbf{x}_{2 n-2}\right)$ given by Peterson's formulae (2).

More generally and with $z_{0}:=0$

$$
\begin{aligned}
& f_{k}\left(z_{k-1}, z_{k}, u^{k}\right):=\sqrt{z_{k}+f_{k}^{2}\left(u^{k}\right)-z_{k-1} \cos ^{2}\left(u^{k}\right)}, \quad k=1, \ldots, n-1, \\
& g_{k}\left(z_{k-1}, z_{k}, u^{k}\right):=\int_{0}^{u^{k}} \frac{\sqrt{f_{k}^{\prime 2}(t)+f_{k}^{2}(t) g_{k}^{\prime 2}(t)-\left(f_{k}^{\prime 2}\left(z_{k-1}, z_{k}, t\right)+z_{k-1} \sin ^{2}(t)\right)}}{f_{k}\left(z_{k-1}, z_{k}, t\right)} d t, \\
& h\left(z_{n-1}, u^{n}\right):=\int_{0}^{u^{n}} \sqrt{h^{\prime 2}(t)-z_{n-1} \sin ^{2}(t)} d t
\end{aligned}
$$

give an $(n-1)$-dimensional family of deformations (8); for $g_{k}\left(u^{k}\right)=0, k=2, \ldots, n-1$ we have $\mathcal{X}_{\mathbf{0}} \subset \mathbb{C}^{n+1}$.

\section{The common conjugate system and non-degenerate joined second fundamental forms}

The fact that $\left(u^{1}, \ldots, u^{n}\right)$ is a conjugate system on $\mathcal{X}_{\mathbf{0}}$ is clear since we have

$$
\partial_{u^{k}} \partial_{u^{j}} \mathcal{X}_{\mathbf{0}}=-\tan \left(u^{j}\right) \partial_{u^{k}} \mathcal{X}_{\mathbf{0}}, \quad 1 \leq k<j \leq n
$$

With the normal field

$$
\hat{N}_{\mathbf{0}}:=\left(\sqrt{a_{0}}\right)^{-1} \mathbf{C}_{0} e_{0}+\sum_{k=1}^{n}\left(\sqrt{a_{k}}\right)^{-1} \mathbf{C}_{k} \sin \left(u^{k}\right) e_{k}
$$

we have $\hat{N}_{\mathbf{0}}^{T} d^{2} \mathcal{X}_{\mathbf{0}}=-\sum_{k=1}^{n} \mathbf{C}_{k}^{2}\left(d u^{k}\right)^{2}$. To see that $\left(u^{1}, \ldots, u^{n}\right)$ is a conjugate system on

$$
\begin{aligned}
\mathcal{X}=\left(x_{0}, \ldots, x_{2 n-2}\right):= & \sum_{k=1}^{n-1} \mathbf{C}_{k} f_{k}\left(u^{k}\right)\left(\cos \left(g_{k}\left(u^{k}\right)\right) e_{2 k-2}+\sin \left(g_{k}\left(u^{k}\right)\right) e_{2 k-1}\right) \\
& +h\left(u^{n}\right) e_{2 n-2}
\end{aligned}
$$

we have again $\partial_{u^{k}} \partial_{u^{j}} \mathcal{X}=-\tan \left(u^{j}\right) \partial_{u^{k}} \mathcal{X}, 1 \leq k<j \leq n$; again the $n-1$ fields

$$
\begin{array}{ll}
\left.\partial_{u^{1}} \mathcal{X}\right|_{u^{1}, u^{2}, \ldots, u^{k}, \ldots, u^{n}=\mathrm{const}}, & \left.\partial_{u^{2}} \mathcal{X}\right|_{u^{1}, u^{2}, \ldots, \widehat{u^{k}}, \ldots, u^{n}=\mathrm{const}}, \quad \ldots, \\
\left.\widehat{\partial_{u^{k}} \mathcal{X}}\right|_{u^{1}, u^{2}, \ldots, u^{k}, \ldots, u^{n}=\mathrm{const}}, \quad \ldots,\left.\quad \partial_{u^{n}} \mathcal{X}\right|_{u^{1}, u^{2}, \ldots, u^{k}, \ldots, u^{n}=\mathrm{const}}, \quad k=1, \ldots, n
\end{array}
$$


generate ruled $n$-dimensional developables in $\mathbb{C}^{2 n-1}$ because the only term producing shape is $\partial_{u^{k}} \partial_{u^{k}} \mathcal{X}$.

For the non-degenerate joined second fundamental forms property we have

$$
\begin{aligned}
& u^{n}=h^{-1}\left(x_{2 n-2}\right), \quad h^{\prime}\left(u^{n}\right) d u^{n}=d x_{2 n-2}, \quad u^{k}=g_{k}^{-1}\left(\tan ^{-1}\left(\frac{x_{2 k-1}}{x_{2 k-2}}\right)\right), \\
& \mathbf{C}_{k}^{2} f_{k}^{2}\left(u^{k}\right) g_{k}^{\prime}\left(u^{k}\right) d u^{k}=x_{2 k-2} d x_{2 k-1}-x_{2 k-1} d x_{2 k-2}, \quad k=1, \ldots, n-1
\end{aligned}
$$

and $\mathcal{X}$ is given implicitly by the zeroes of the functionally independent

$$
F_{k}:=\left(x_{2 k-2}\right)^{2}+\left(x_{2 k-1}\right)^{2}-\mathbf{C}_{k}^{2} f_{k}^{2}\left(u^{k}\right), \quad k=1, \ldots, n-1 .
$$

We have the natural linearly independent normal fields

$$
\begin{aligned}
N_{k}:= & \nabla F_{k}=2\left(x_{2 k-2} e_{2 k-2}+x_{2 k-1} e_{2 k-1}\right)-\frac{2 f_{k}^{\prime}\left(u^{k}\right)\left(-x_{2 k-1} e_{2 k-2}+x_{2 k-2} e_{2 k-1}\right)}{f_{k}\left(u^{k}\right) g_{k}^{\prime}\left(u^{k}\right)} \\
& +2 \mathbf{C}_{k}^{2} f_{k}^{2}\left(u^{k}\right)\left[\sum_{j=k+1}^{n-1} \frac{\tan \left(u^{j}\right)\left(-x_{2 j-1} e_{2 j-2}+x_{2 j-2} e_{2 j-1}\right)}{\mathbf{C}_{j}^{2} f_{j}^{2}\left(u^{j}\right) g_{j}^{\prime}\left(u^{j}\right)}+\frac{\tan \left(u^{n}\right) e_{2 n-2}}{h^{\prime}\left(u^{n}\right)}\right], \\
& k=1, \ldots, n-1,
\end{aligned}
$$

and

$$
\begin{aligned}
\partial_{u^{l}} \partial_{u^{l}} \mathcal{X}= & -\sum_{j=1}^{l-1}\left(x_{2 j-2} e_{2 j-2}+x_{2 j-1} e_{2 j-1}\right)+\left(\frac{f_{l}^{\prime \prime}\left(u^{l}\right)}{f_{l}\left(u^{l}\right)}-g_{l}^{\prime 2}\left(u^{l}\right)\right)\left(x_{2 l-2} e_{2 l-2}+x_{2 l-1} e_{2 l-1}\right) \\
& +g_{l}^{\prime}\left(u^{l}\right)\left(\frac{2 f_{l}^{\prime}\left(u^{l}\right)}{f_{l}\left(u^{l}\right)}+\frac{g_{l}^{\prime \prime}\left(u^{l}\right)}{g_{l}^{\prime}\left(u^{l}\right)}\right)\left(-x_{2 l-1} e_{2 l-2}+x_{2 l-2} e_{2 l-1}\right), \quad l=1, \ldots, n-1, \\
\partial_{u^{n}} \partial_{u^{n}} \mathcal{X}= & -\sum_{l=1}^{n-1}\left(x_{2 l-2} e_{2 l-2}+x_{2 l-1} e_{2 l-1}\right)+h^{\prime \prime}\left(u^{n}\right) e_{2 n-2},
\end{aligned}
$$

and the second fundamental form

$$
\begin{aligned}
N_{k}^{T} d^{2} \mathcal{X}= & 2 \mathbf{C}_{k}^{2} f_{k}^{2}\left[\left(\frac{f_{k}^{\prime \prime}\left(u^{k}\right)}{f_{k}\left(u^{k}\right)}-g_{k}^{\prime 2}\left(u^{k}\right)-\frac{f_{k}^{\prime}\left(u^{k}\right)}{f_{k}\left(u^{k}\right)}\left(\frac{2 f_{k}^{\prime}\left(u^{k}\right)}{f_{k}\left(u^{k}\right)}+\frac{g_{k}^{\prime \prime}\left(u^{k}\right)}{g_{k}^{\prime}\left(u^{k}\right)}\right)\right)\left(d u^{k}\right)^{2}\right. \\
& +\sum_{l=k+1}^{n-1}\left(\tan \left(u^{l}\right)\left(\frac{2 f_{l}^{\prime}\left(u^{l}\right)}{f_{l}\left(u^{l}\right)}+\frac{g_{l}^{\prime \prime}\left(u^{l}\right)}{g_{l}^{\prime}\left(u^{l}\right)}\right)-1\right)\left(d u^{l}\right)^{2} \\
& \left.+\left(\frac{\tan \left(u^{n}\right) h^{\prime \prime}\left(u^{n}\right)}{h^{\prime}\left(u^{n}\right)}-1\right)\left(d u^{n}\right)^{2}\right], \quad k=1, \ldots, n-1 .
\end{aligned}
$$

For Peterson's deformations of higher dimensional quadrics we have

$$
N_{k}^{T} d^{2} \mathcal{X}=-2 a_{0} \mathbf{C}_{k}^{2} f_{k}^{2}\left(\frac{a_{k} z_{k-1}\left(d u^{k}\right)^{2}}{g_{k}^{\prime 2}\left(u^{k}\right) f_{k}^{4}\left(u^{k}\right)}+\sum_{l=k+1}^{n-1} \frac{a_{l}\left(z_{l-1}-z_{l}\right)\left(d u^{l}\right)^{2}}{g_{l}^{\prime 2}\left(u^{l}\right) f_{l}^{4}\left(u^{l}\right)}+\frac{a_{n}\left(d u^{n}\right)^{2}}{a_{0} h^{\prime 2}\left(u^{n}\right)}\right)
$$

It is now enough to check the open non-degenerate joined second fundamental forms property 
only for $\mathbf{z}=(1,1, \ldots, 1)$. Thus with $\delta:=\frac{a_{n}}{a_{0} \sin ^{2}\left(u^{n}\right)+a_{n} \cos ^{2}\left(u^{n}\right)}$ we need

$$
0 \neq\left|\begin{array}{cccccc}
\mathbf{C}_{1} & \mathbf{C}_{2} & \mathbf{C}_{3} & \ldots & \mathbf{C}_{n-1} & \delta^{-1} \mathbf{C}_{n} \\
\frac{a_{1}}{a_{1}-a_{0}} & 0 & 0 & \cdots & 0 & \sin ^{2}\left(u^{1}\right) \\
0 & \frac{a_{2}}{a_{2}-a_{0}} & 0 & \cdots & 0 & \sin ^{2}\left(u^{2}\right) \\
0 & 0 & \frac{a_{3}}{a_{3}-a_{0}} & \cdots & 0 & \sin ^{2}\left(u^{3}\right) \\
\vdots & \vdots & \vdots & \cdots & \vdots & \vdots \\
0 & 0 & 0 & \cdots & \frac{a_{n-1}}{a_{n-1}-a_{0}} & \sin ^{2}\left(u^{n-1}\right)
\end{array}\right|
$$

almost everywhere, which is straightforward.

Remark 3. Note that a-priori $\mathcal{X}_{\mathbf{1}}$ comes close to lie in a degenerate deformation of $\mathbb{C}^{n+1}$ in

$\mathbb{C}^{2 n-1}: \hat{N}_{\mathbf{0}}^{T} d^{2} \mathcal{X}_{\mathbf{0}}-\left(\sum_{k=1}^{n-1} \frac{1}{2 a_{k}} N_{k}\right)^{T} d^{2} \mathcal{X}_{\mathbf{1}}$ depends only on $\left(d u^{n}\right)^{2}$ and this is as closest to 0 as we can get.

\section{Conjugate systems}

Consider the complexified Euclidean space

$$
\left(\mathbb{C}^{n},\langle\cdot, \cdot\rangle\right), \quad\langle x, y\rangle:=x^{T} y, \quad|x|^{2}:=x^{T} x, \quad x, y \in \mathbb{C}^{n}
$$

with standard basis $\left\{e_{j}\right\}_{j=1, \ldots, n}, e_{j}^{T} e_{k}=\delta_{j k}$.

Isotropic (null) vectors are those vectors $v$ of length $0\left(|v|^{2}=0\right)$; since most vectors are not isotropic we shall call a vector simply vector and we shall only emphasize isotropic when the vector is assumed to be isotropic. The same denomination will apply in other settings: for example we call quadric a non-degenerate quadric (a quadric projectively equivalent to the complex unit sphere).

For $n \geq 3$ consider the $n$-dimensional sub-manifold

$$
x=x\left(u^{1}, u^{2}, \ldots, u^{n}\right) \subset \mathbb{C}^{n+p}, \quad d u^{1} \wedge d u^{2} \wedge \cdots \wedge d u^{n} \neq 0
$$

such that the tangent space at any point of $x$ is not isotropic (the scalar product induced on it by the Euclidean one on $\mathbb{C}^{n+p}$ is not degenerate; this assures the existence of orthonormal normal frames). We shall always have Latin indices $j, k, l, m, p, q \in\{1, \ldots, n\}$, Greek ones $\alpha, \beta, \gamma \in\{n+1, \ldots, n+p\}$ and mute summation for upper and lower indices when clear from the context; also we shall preserve the classical notation $d^{2}$ for the tensorial (symmetric) second derivative. We have the normal frame $N:=\left[\begin{array}{llll}N_{n+1} & \ldots & N_{n+p}\end{array}\right], N^{T} N=I_{p}$, the first $|d x|^{2}=$ $g_{j k} d u^{j} \odot d u^{k}$ and second $d^{2} x^{T} N=\left[h_{j k}^{n+1} d u^{j} \odot d u^{k} \ldots h_{j k}^{n+p} d u^{j} \odot d u^{k}\right]$ fundamental forms, the Christoffel symbols $\Gamma_{j k}^{l}=\frac{g^{l m}}{2}\left[\partial_{u^{k}} g_{j m}+\partial_{u^{j}} g_{k m}-\partial_{u^{m}} g_{j k}\right]$, the Riemann curvature $R_{j m k l}=$ $g_{m p} R_{j k l}^{p}=g_{m p}\left[\partial_{u^{l}} \Gamma_{j k}^{p}-\partial_{u^{k}} \Gamma_{j l}^{p}+\Gamma_{j k}^{q} \Gamma_{q l}^{p}-\Gamma_{j l}^{q} \Gamma_{q k}^{p}\right]$ tensor, the normal connection $N^{T} d N=$ $\left\{n_{\beta j}^{\alpha} d u^{j}\right\}_{\alpha, \beta=n+1, \ldots, n+p}, n_{\beta j}^{\alpha}=-n_{\alpha j}^{\beta}$ and the curvature $r_{\alpha j k}^{\beta}=\partial_{u^{k}} n_{\alpha j}^{\beta}-\partial_{u^{j}} n_{\alpha k}^{\beta}+n_{\alpha j}^{\gamma} n_{\gamma k}^{\beta}-n_{\alpha k}^{\gamma} n_{\gamma j}^{\beta}$ tensor of the normal bundle.

We have the Gauß-Weingarten (GW) equations

$$
\partial_{u^{k}} \partial_{u^{j}} x=\Gamma_{j k}^{l} \partial_{u^{\imath}} x+h_{j k}^{\alpha} N_{\alpha}, \quad \partial_{u^{j}} N_{\alpha}=-h_{j k}^{\alpha} g^{k l} \partial_{u^{\imath}} x+n_{j \alpha}^{\beta} N_{\beta}
$$

and their integrability conditions $\partial_{u^{l}}\left(\partial_{u^{k}} \partial_{u^{j}} x\right)=\partial_{u^{k}}\left(\partial_{u^{l}} \partial_{u^{j}} x\right), \partial_{u^{k}}\left(\partial_{u^{j}} N_{\alpha}\right)=\partial_{u^{j}}\left(\partial_{u^{k}} N_{\alpha}\right)$, from where one obtains by taking the tangential and normal components (using $-\partial_{u} g^{j k}=$ 
$g^{j m} \Gamma_{m l}^{k}+g^{k m} \Gamma_{m l}^{j}$ and the GW equations themselves) the Gauß-Codazzi-Mainardi(-Peterson)Ricci (G-CMP-R) equations

$$
\begin{aligned}
& R_{j m k l}=\sum_{\alpha}\left(h_{j k}^{\alpha} h_{l m}^{\alpha}-h_{j l}^{\alpha} h_{k m}^{\alpha}\right), \\
& \partial_{u^{l}} h_{j k}^{\alpha}-\partial_{u^{k}} h_{j l}^{\alpha}+\Gamma_{j k}^{m} h_{m l}^{\alpha}-\Gamma_{j l}^{m} h_{m k}^{\alpha}+h_{j k}^{\beta} n_{\beta l}^{\alpha}-h_{j l}^{\beta} n_{\beta k}^{\alpha}=0, \\
& r_{\alpha j k}^{\beta}=h_{j l}^{\alpha} g^{l m} h_{m k}^{\beta}-h_{k l}^{\alpha} g^{l m} h_{m j}^{\beta} .
\end{aligned}
$$

If we have conjugate system $h_{j k}^{\alpha}=: \delta_{j k} h_{j}^{\alpha}$, then the above equations become:

$$
\begin{aligned}
& R_{j k j k}=-R_{j k k j}=\sum_{\alpha} h_{j}^{\alpha} h_{k}^{\alpha}, \quad \partial_{u^{k}} h_{j}^{\alpha}=\Gamma_{j k}^{j} h_{j}^{\alpha}-\Gamma_{j j}^{k} h_{k}^{\alpha}-h_{j}^{\beta} \eta_{\beta k}^{\alpha}, \quad j \neq k, \\
& R_{j k l m}=0 \quad \text { otherwise }, \\
& \Gamma_{j k}^{l} h_{l}^{\alpha}=\Gamma_{j l}^{k} h_{k}^{\alpha}, \quad j, k, l \text { distinct, } \quad r_{\alpha j k}^{\beta}=\left(h_{j}^{\alpha} h_{k}^{\beta}-h_{j}^{\beta} h_{k}^{\alpha}\right) g^{j k} .
\end{aligned}
$$

In particular for lines of curvature parametrization $\left(g_{j k}=\delta_{j k} g_{j k}\right)$ we have flat normal bundle, so one can choose up to multiplication on the right by a constant matrix $\in \mathbf{O}_{p}(\mathbb{C})$ normal frame $N$ with zero normal connection $N^{T} d N=0$.

This constitutes a differential system in the $n p$ unknowns $h_{j}^{\alpha}$ and the yet to be determined coefficients $\eta_{\beta k}^{\alpha}$; according to Cartan's exterior differential systems in involution tools in order to study deformations of $n$-dimensional sub-manifolds of $\mathbb{C}^{n+p}$ in conjugate system parameterization one must iteratively apply compatibility conditions (commuting of mixed derivatives) to the equations of this system and their algebraic-differential consequences, introducing new variables as necessary and assuming only identities obtained at previous iterations and general identities for the Riemann curvature tensor (symmetries and Bianchi identities):

$$
\begin{aligned}
& R_{j k l m}=-R_{k j l m}=-R_{j k m l}=R_{l m j k}, \quad R_{j k l m}+R_{j l m k}+R_{j m k l}=0, \\
& R_{j k l m ; q}+R_{j k m q ; l}+R_{j k q l ; m}=0, \\
& R_{j k l m ; q}:=\partial_{u^{q}} R_{j k l m}-\Gamma_{q j}^{r} R_{r k l m}-\Gamma_{q k}^{r} R_{j r l m}-\Gamma_{q l}^{r} R_{j k r m}-\Gamma_{q m}^{r} R_{j k l r}
\end{aligned}
$$

until no further conditions appear from compatibility conditions. However one cannot use in full the Cartan's exterior differential forms and moving frames tools (see, e.g. [1]), since they are best suited for arbitrary (orthonormal) tangential frames and orthonormal normal ones and their corresponding change of frames; thus one loses the advantage of special coordinates suited to our particular problem.

In our case we only obtain

$$
\begin{aligned}
& \partial_{u^{l}} R_{j k j k}=\left(\Gamma_{j l}^{j}+\Gamma_{k l}^{k}\right) R_{j k j k}-\Gamma_{k k}^{l} R_{j l j l}-\Gamma_{j j}^{l} R_{k l k l}, \quad j, k, l \text { distinct, } \\
& \Gamma_{l k}^{m} R_{j m j m}-\Gamma_{m k}^{l} R_{j l j l}=0, \quad j, k, l, m \text { distinct. }
\end{aligned}
$$

Remark 4. Differentiating the first equations of (10) with respect to $u^{l}, l \neq j, k$ and using (10) itself we obtain

$$
\begin{aligned}
\partial_{u^{l}} R_{j k j k} & =\sum_{\alpha}\left(\partial_{u^{l}} h_{j}^{\alpha} h_{k}^{\alpha}+h_{j}^{\alpha} \partial_{u^{l}} h_{k}^{\alpha}\right) \\
& =\sum_{\alpha}\left[\left(\Gamma_{j l}^{j} h_{j}^{\alpha}-\Gamma_{j j}^{l} h_{l}^{\alpha}-h_{j}^{\beta} \eta_{\beta l}^{\alpha}\right) h_{k}^{\alpha}+h_{j}^{\alpha}\left(\Gamma_{k l}^{k} h_{k}^{\alpha}-\Gamma_{k k}^{l} h_{l}^{\alpha}-h_{k}^{\beta} \eta_{\beta l}^{\alpha}\right)\right] \\
& =\left(\Gamma_{j l}^{j}+\Gamma_{k l}^{k}\right) R_{j k j k}-\Gamma_{k k}^{l} R_{j l j l}-\Gamma_{j j}^{l} R_{k l k l},
\end{aligned}
$$

that is the first equations of (11), so the covariant derivative of the Gauß equations become, via the G-CMP equations, the Bianchi second identity (the second equations of (11) being consequence of the CMP equations is obvious; see also [1]). 


\section{The non-degenerate joined second fundamental forms assumption}

With an eye towards our interests (deformations in $\mathbb{C}^{2 n-1}$ of quadrics in $\mathbb{C}^{n+1}$ and with common conjugate system) we make the genericity assumption of non-degenerate joined second fundamental forms of $x_{0}, x$ : with $d^{2} x_{0}^{T} N_{0}=: h_{j}^{0}\left(d u^{j}\right)^{2}$ being the second fundamental form of the quadric $x_{0} \subset \mathbb{C}^{n+1}$ whose deformation $x \subset \mathbb{C}^{2 n-1}$ is (that is $\left|d x_{0}\right|^{2}=|d x|^{2}$ ) the vectors $h_{j}:=\left[\begin{array}{llll}i h_{j}^{0} & h_{j}^{n+1} & \ldots & h_{j}^{2 n-1}\end{array}\right]^{T}$ are linearly independent. From the Gauß equations we obtain $h_{j}^{0} h_{k}^{0}=R_{j k j k}=\sum_{\alpha} h_{j}^{\alpha} h_{k}^{\alpha}, j \neq k \Leftrightarrow h_{j}^{T} h_{k}=\delta_{j k}\left|h_{j}\right|^{2}$; thus the vectors $h_{j} \subset \mathbb{C}^{n}$ are further orthogonal, which prevents them from being isotropic (should one of them be isotropic, by a rotation of $\mathbb{C}^{n}$ one can make it $f_{1}$ and after subtracting suitable multiples of $f_{1}$ from the remaining ones by another rotation of $\mathbb{C}^{n}$ the remaining ones linear combinations of $e_{3}, \ldots, e_{n}$, so we would have $n-1$ linearly independent orthogonal vectors in $\mathbb{C}^{n-2}$, a contradiction), so $\mathbf{a}_{j}:=\left|h_{j}\right| \neq 0$, $h_{j}=: \mathbf{a}_{j} v_{j}, R:=\left[\begin{array}{lll}v_{1} & \ldots & v_{n}\end{array}\right] \subset \mathbf{O}_{n}(\mathbb{C})$.

Thus with $\eta_{0 j}^{\alpha}=-\eta_{\alpha j}^{0}:=0,\left(\eta_{\beta j}^{\alpha}\right)_{\alpha, \beta=0, n+1, \ldots, 2 n-1}=: \Upsilon_{j}=-\Upsilon_{j}^{T}$ we have reduced the problem to finding $R=\left[\begin{array}{lll}v_{1} & \ldots & v_{n}\end{array}\right] \subset \mathbf{O}_{n}(\mathbb{C}), \mathbf{a}_{j} \subset \mathbb{C}^{*}, \Upsilon_{j} \subset \mathbf{M}_{n}(\mathbb{C}), \Upsilon_{j}=-\Upsilon_{j}^{T}, \Upsilon_{j} e_{1}=0$ satisfying the differential system

$$
\begin{aligned}
& \partial_{u^{k}} \log \mathbf{a}_{j}=\Gamma_{j k}^{j}, \quad \partial_{u^{k}} v_{j}=-\Gamma_{j j}^{k} \frac{\mathbf{a}_{k}}{\mathbf{a}_{j}} v_{k}-\Upsilon_{k} v_{j}, \\
& \partial_{u^{k}} \Upsilon_{j}-\partial_{u^{j}} \Upsilon_{k}-\left[\Upsilon_{j}, \Upsilon_{k}\right]=-g^{j k} \mathbf{a}_{j} \mathbf{a}_{k}\left(I_{n}-e_{1} e_{1}^{T}\right)\left(v_{j} v_{k}^{T}-v_{k} v_{j}^{T}\right)\left(I_{n}-e_{1} e_{1}^{T}\right), \quad j \neq k, \\
& \sum_{j} \frac{\left(h_{j}^{0}\right)^{2}}{\mathbf{a}_{j}^{2}}+1=0
\end{aligned}
$$

derived from the CMP-R equations and $\mathbf{a}_{j} v_{j}^{1}=i h_{j}^{0}, \sum_{j}\left(v_{j}^{1}\right)^{2}=1$ and with the linear element further satisfying the condition

$$
\Gamma_{j k}^{l}=0, \quad j, k, l \text { distinct }
$$

derived from the CMP equations.

First we shall investigate the consequences of (13), via the properties of the Riemann curvature tensor, on the other Christoffel symbols. For $j, k, l$ distinct we have $0=g^{p m} R_{j m k l}=$ $R_{j k l}^{p}=\partial_{u^{l}} \Gamma_{j k}^{p}-\partial_{u^{k}} \Gamma_{j l}^{p}+\Gamma_{j k}^{q} \Gamma_{q l}^{p}-\Gamma_{j l}^{q} \Gamma_{q k}^{p}$; thus for $p=k$ we obtain

$$
\partial_{u l} \Gamma_{k j}^{k}=\Gamma_{k l}^{k} \Gamma_{l j}^{l}+\Gamma_{k j}^{k} \Gamma_{j l}^{j}-\Gamma_{k l}^{k} \Gamma_{k j}^{k}, \quad j, k, l \text { distinct. }
$$

We also have $R_{j j l}^{p}=g^{p m} R_{j m j l}=g^{p l} R_{j l j l}, j \neq l$, so

$$
\begin{aligned}
& g^{p l} R_{j l j l}=\partial_{u^{l}} \Gamma_{j j}^{p}+\Gamma_{j j}^{p}\left(\Gamma_{p l}^{p}-\Gamma_{j l}^{j}\right)+\Gamma_{j j}^{l} \Gamma_{l l}^{p}, \quad j, l, p \text { distinct, } \\
& g^{j l} R_{j l j l}=\partial_{u^{l}} \Gamma_{j j}^{j}-\partial_{u^{j}} \Gamma_{j l}^{j}+\Gamma_{j j}^{l} \Gamma_{l l}^{j}-\Gamma_{l j}^{l} \Gamma_{j l}^{j}, \\
& g^{l l} R_{j l j l}=\partial_{u^{l}} \Gamma_{j j}^{l}-\partial_{u^{j}} \Gamma_{l j}^{l}+\Gamma_{j j}^{q} \Gamma_{l q}^{l}-\Gamma_{j l}^{j} \Gamma_{j j}^{l}-\left(\Gamma_{l j}^{l}\right)^{2}, \quad j \neq l .
\end{aligned}
$$

Conversely, (13) and (14) imply $R_{j k l m}=0$ for three of $j, k, l, m$ distinct.

Remark 5. Note that (13) are valid for orthogonal coordinates, so conjugate systems with the property (13) are a natural projective generalization of lines of curvature on $n$-dimensional sub-manifolds $x \subset \mathbb{C}^{n+p}$ (to see this first $\partial_{u^{k}} \partial_{u^{j}} x=\Gamma_{j k}^{j} \partial_{u^{j}} x+\Gamma_{k j}^{k} \partial_{u^{k}} x, j \neq k$ is affine invariant (thus $\Gamma_{j k}^{j}, \Gamma_{k j}^{k}$ are also affine invariants) and further $\partial_{u^{k}} \partial_{u^{j}} \frac{x}{\rho}=\left(\Gamma_{j k}^{j}-\partial_{u^{k}} \log \rho\right) \partial_{u^{j}} \frac{x}{\rho}+\left(\Gamma_{k j}^{k}-\right.$ $\left.\partial_{u^{j}} \log \rho\right) \partial_{u^{k}} \frac{x}{\rho}, j \neq k$ for $\rho \subset \mathbb{C}^{*}$ with $\left.\partial_{u^{k}} \partial_{u^{j}} \rho=\Gamma_{j k}^{j} \partial_{u^{j}} \rho+\Gamma_{k j}^{k} \partial_{u^{k}} \rho, j \neq k\right)$. 
Imposing the compatibility conditions

$$
\partial_{u^{l}}\left(\partial_{u^{k}} \log \mathbf{a}_{j}\right)=\partial_{u^{k}}\left(\partial_{u^{l}} \log \mathbf{a}_{j}\right), \quad \partial_{u^{l}}\left(\partial_{u^{k}} v_{j}\right)=\partial_{u^{k}}\left(\partial_{u^{l}} v_{j}\right), \quad j, k, l \text { distinct }
$$

we obtain

$$
\partial_{u^{l}} \Gamma_{j k}^{j}-\partial_{u^{k}} \Gamma_{j l}^{j}=0=\partial_{u^{l}} \Gamma_{j j}^{k}+\Gamma_{j j}^{k}\left(\Gamma_{k l}^{k}-\Gamma_{j l}^{j}\right)+\Gamma_{j j}^{l} \Gamma_{l l}^{k}-g^{k l} h_{j}^{0} h_{l}^{0}, \quad j, k, l \text { distinct },
$$

which are consequences of (14) and the first equations of (15).

From the first equations of (12) we get by integration a precise determination of $\mathbf{a}_{j}$ up to multiplication by a function of $u^{j}$; thus the $\mathbf{a}_{j}$ part of the solution depends on at most $n$ functions of one variable (with the last equation of (12) also to be taken into consideration); we shall see later that the remaining part of the differential system involves the normal bundle and its indeterminacy, so it will not produce a bigger space of solutions (no functional information is allowed in the normal bundle). From the CMP equations of $x_{0}$ and the second equations of (16) we obtain with $\gamma_{j k}:=\Gamma_{j j}^{k} \frac{h_{k}^{0}}{h_{j}^{0}}, j \neq k$ :

$$
\partial_{u^{l}} \gamma_{j k}=\gamma_{j k} \gamma_{j l}-\gamma_{j l} \gamma_{l k}-\gamma_{j k} \gamma_{k l}+g^{k l} h_{k}^{0} h_{l}^{0}, \quad j, k, l \text { distinct. }
$$

From the first equations of (12) and the CMP equations of $x_{0}$ we obtain with $\mathbf{b}_{j}:=\frac{h_{j}^{0}}{\mathbf{a}_{j}}$

$$
\partial_{u^{k}} \log \mathbf{b}_{j}=-\gamma_{j k}, \quad j \neq k,
$$

so differentiating the last equation of (12) we obtain

$$
\partial_{u^{j}} \log \mathbf{b}_{j}=\mathbf{b}_{j}^{-2} \sum_{l \neq j} \mathbf{b}_{l}^{2} \gamma_{l j}
$$

This assures that

$$
\Upsilon_{k}:=-\partial_{u^{k}} R R^{T}-\sum_{j \neq k} \gamma_{j k} \frac{\mathbf{b}_{j}}{\mathbf{b}_{k}}\left(v_{k} v_{j}^{T}-v_{j} v_{k}^{T}\right)
$$

satisfies $e_{1}^{T} \Upsilon_{k}=0$. Thus we have reduced the problem to finding $\mathbf{b}_{j}$ satisfying (18), (19) (in this case $\sum_{j} \mathbf{b}_{j}^{2}+1=0$ becomes a prime integral of (18), (19) and removes a constant from the space of solutions) and then completing $v_{j}^{1}=-i \mathbf{b}_{j}$ to $R=\left[v_{1} \ldots v_{n}\right] \subset \mathbf{O}_{n}(\mathbb{C})$ in an arbitrary manner (that is undetermined up to multiplication on the left with $R^{\prime} \subset \mathbf{O}_{n}(\mathbb{C}), R^{\prime} e_{1}=e_{1}$ ); with the second fundamental form of $x$ found one finds $x$ by the integration of a Ricatti equation and quadratures (the Gauß-Bonnet(-Peterson) theorem). $\Upsilon_{j}$ given by (20) will satisfy

$$
\partial_{u^{k}} \Upsilon_{j}-\partial_{u^{j}} \Upsilon_{k}-\left[\Upsilon_{j}, \Upsilon_{k}\right]=-\frac{g^{j k} h_{j}^{0} h_{k}^{0}}{\mathbf{b}_{j} \mathbf{b}_{k}}\left(I_{n}-e_{1} e_{1}^{T}\right)\left(v_{j} v_{k}^{T}-v_{k} v_{j}^{T}\right)\left(I_{n}-e_{1} e_{1}^{T}\right), \quad j \neq k .
$$

Imposing the compatibility condition $\partial_{u^{k}}\left(\partial_{u^{j}} \log \mathbf{b}_{j}\right)=\partial_{u^{j}}\left(\partial_{u^{k}} \log \mathbf{b}_{j}\right), j \neq k$ on (18), (19) we obtain

$$
\partial_{u^{k}}\left(\gamma_{k j} \frac{\mathbf{b}_{k}}{\mathbf{b}_{j}}\right)+\partial_{u^{j}}\left(\gamma_{j k} \frac{\mathbf{b}_{j}}{\mathbf{b}_{k}}\right)-\sum_{l \neq j, k}\left(\gamma_{l j} \gamma_{l k}-g^{j k} h_{j}^{0} h_{k}^{0}\right) \frac{\mathbf{b}_{l}^{2}}{\mathbf{b}_{j} \mathbf{b}_{k}}=0, \quad j \neq k .
$$

Now (21) becomes

$$
\begin{aligned}
& \sum_{m \neq j} \partial_{u^{k}}\left(\gamma_{m j} \frac{\mathbf{b}_{m}}{\mathbf{b}_{j}}\right)\left(v_{j} v_{m}^{T}-v_{m} v_{j}^{T}\right)-\sum_{l \neq k} \partial_{u^{j}}\left(\gamma_{l k} \frac{\mathbf{b}_{l}}{\mathbf{b}_{k}}\right)\left(v_{k} v_{l}^{T}-v_{l} v_{k}^{T}\right) \\
& \quad+\sum_{\substack{m \neq j, l \neq k\\
}} \gamma_{m j} \gamma_{l k} \frac{\mathbf{b}_{m} \mathbf{b}_{l}}{\mathbf{b}_{j} \mathbf{b}_{k}}\left[\delta_{m k}\left(v_{j} v_{l}^{T}-v_{l} v_{j}^{T}\right)+\delta_{m l}\left(v_{k} v_{j}^{T}-v_{j} v_{k}^{T}\right)-\delta_{j l}\left(v_{k} v_{m}^{T}-v_{m} v_{k}^{T}\right)\right] \\
& \quad=\frac{g^{j k} h_{j}^{0} h_{k}^{0}}{\mathbf{b}_{j} \mathbf{b}_{k}}\left(I_{n}-e_{1} e_{1}^{T}\right)\left(v_{j} v_{k}^{T}-v_{k} v_{j}^{T}\right)\left(I_{n}-e_{1} e_{1}^{T}\right), \quad j \neq k,
\end{aligned}
$$


which boils down to (22) and

$$
\partial_{u^{k}}\left(\gamma_{l j} \frac{\mathbf{b}_{l}}{\mathbf{b}_{j}}\right)+\left(\gamma_{l k} \gamma_{k j}-g^{j k} h_{j}^{0} h_{k}^{0}\right) \frac{\mathbf{b}_{l}}{\mathbf{b}_{j}}=0, \quad j, k, l \text { distinct },
$$

which follows from (17) and (18).

Note that (22) can be written as

$$
\begin{aligned}
& \mathbf{b}_{j}^{2}\left(\partial_{u^{j}} \gamma_{j k}+2 \gamma_{j k} \gamma_{k j}\right)+\mathbf{b}_{k}^{2}\left(\partial_{u^{k}} \gamma_{k j}+2 \gamma_{k j} \gamma_{j k}\right) \\
& \quad+\sum_{l \neq j, k} \mathbf{b}_{l}^{2}\left(\partial_{u^{k}} \gamma_{l j}+2\left(\gamma_{l k} \gamma_{k j}+\gamma_{l j} \gamma_{j k}-\gamma_{l j} \gamma_{l k}\right)\right)=0, \quad j \neq k,
\end{aligned}
$$

so the differential system (18), (19) is in involution (completely integrable) for

$$
\partial_{u^{j}} \gamma_{j k}=\partial_{u^{k}} \gamma_{k j}=-2 \gamma_{j k} \gamma_{k j}, \quad \partial_{u^{k}} \gamma_{l j}=2\left(\gamma_{l j} \gamma_{l k}-\gamma_{l k} \gamma_{k j}-\gamma_{l j} \gamma_{j k}\right), \quad j, k, l \text { distinct. (23) }
$$

Thus if (23) holds, then the solution of (18), (19) is obtained by integrating $n$ first order ODE's (namely finding the functions of $u^{j}$ upon whose multiplication with $\mathbf{a}_{j}$ depends), so the space of solutions depends on $(n-1)$ constants (the prime integral $\sum_{j} \mathbf{b}_{j}^{2}+1=0$ removes a constant from the space of solutions); if (23) does not hold, then the space of solutions depends on less than $(n-1)$ constants. Since for Peterson's deformations of higher dimensional quadrics (or more generally for deformations of sub-manifolds of the type (8) with $f_{k}, g_{k}, h$ given by (9) with $\left.g_{k}\left(u^{k}\right)=0, k=2, \ldots, n-1\right)$ we already have an $(n-1)$-dimensional explicit family of deformations, we conclude that this family is maximal and that (23) holds in these cases.

Remark 6. Note that (23) generalizes the case $n=2$ condition $\partial_{u^{1}} \gamma_{12}=\partial_{u^{2}} \gamma_{21}=-2 \gamma_{12} \gamma_{21}$ that the conjugate system $\left(u^{1}, u^{2}\right)$ is common to a Peterson's 1-dimensional family of deformations of surfaces (see Bianchi [2, Vol. 2, $\S \S 294,295]$ ), so conjugate systems of $n$-dimensional submanifolds in $\mathbb{C}^{n+1}$ satisfying (13) and (23) are a natural generalization of Peterson's approach in the deformation problem.

\section{Acknowledgements}

I would like to thank the referees for useful suggestions. The research has been supported by the University of Bucharest.

\section{References}

[1] Berger E., Bryant R.L., Griffiths P.A., The Gauss equations and rigidity of isometric embeddings, Duke Math. J. 50 (1983), 803-892.

[2] Bianchi L., Lezioni di geometria differenziale, Vols. 1-4, Nicola Zanichelli Editore, Bologna, 1922, 1923, 1924, 1927.

[3] Calapso P., Intorno alle superficie applicabili sulle quadriche ed alle loro transformazioni, Annali di Mat. 19 (1912), no. 1, 61-82.

Calapso P., Intorno alle superficie applicabili sulle quadriche ed alle loro transformazioni, Annali di Mat. 19 (1912), no. 1, 107-157.

[4] Cartan É., Sur les variétés de courboure constante d'un espace euclidien ou non-euclidien, Bull. Soc. Math. France 47 (1919), 125-160.

Cartan É., Sur les variétés de courboure constante d'un espace euclidien ou non-euclidien, Bull. Soc. Math. France 48 (1920), 132-208.

[5] Darboux G., Leçons sur la théorie générale des surfaces et les applications géométriques du calcul infinitésimal, Vols. 1-4, Gauthier-Villars, Paris, 1894-1917.

[6] Horn R.A., Johnson C.R., Matrix analysis, Cambridge University Press, Cambridge, 1985.

[7] Peterson K.-M., Sur la déformation des surfaces du second ordre, Ann. Fac. Sci. Toulouse Sér. 27 (1905), no. 1, 69-107. 